\title{
On the structure of étale motivic cohomology.
}

\author{
Thomas H. Geisser \\ September 9, 2016 \\ Dedicated to C. Weibel on his 65th birthday

\begin{abstract}
We discuss the structure of integral étale motivic cohomology groups of smooth and projective schemes over algebraically closed fields, finite fields, local fields, and arithmetic schemes.
\end{abstract}

\section{Introduction}

Let $B$ be the spectrum of a Dedekind ring or field, $X$ a smooth and projective variety over $B$, and $\mathbb{Z}(n)$ Bloch's cycle complex. The goal of this paper is to find structure results for the integral étale motivic cohomology groups $H_{\text {et }}^{i}(X, \mathbb{Z}(n))$ of $X$. Our first result concerns algebraically closed base fields:

Theorem 1.1. Let $B=$ Spec $k$ be the spectrum of an algebraically closed field of characteristic $p \geq 0, i \neq 2 n$, and $d=\operatorname{dim} X$. Then we have an isomorphism

$$
H_{\mathrm{et}}^{i}(X, \mathbb{Z}(n)) \cong D^{i}(n) \oplus C^{i}(n) \oplus P^{i}(n),
$$

where

1. $D^{i}(n)$ is uniquely divisible, and vanishes for $i>\min \{2 n, n+d\}$,

2. the prime-to-p torsion part $C^{i}(n)$ is isomorphic to $\mathbb{Q} / \mathbb{Z}\left[\frac{1}{p}\right]^{r} \oplus F^{i}(n)$, invariant under extensions of algebraically closed fields, $F^{i}(n)=\prod_{l \neq p}$ Tor $H_{\mathrm{et}}^{i}\left(X, \mathbb{Z}_{l}\right)$ is a finite group independent of $n, r \in \mathbb{N}_{0}$ is independent of $n$ if $n \neq \frac{i-1}{2}$, and $C^{i}(n)$ vanishes unless $1 \leq i \leq 2 d+1$,

3. the p-primary torsion part $P^{i}(n)$ is isomorphic to $\left(\mathbb{Q}_{p} / \mathbb{Z}_{p}\right)^{s} \oplus P_{c}^{i}(n)$, with $P_{c}^{i}(n)$ an extension of a finite group $F_{p}^{i}(n)$ by a finitely generated torsion $W(k)$-module $U^{i}(n)$, and $P^{i}(n)=0$ unless $n+1 \leq i \leq n+d+1$ and $0 \leq n \leq d$. 
We have the following duality statements:

Proposition 1.2. Under the hypothesis of the theorem,

1. $F^{i}(n)$ and $F_{p}^{i}(n)$ are Pontrjagin dual to $F^{2 d+1-i}(d-n)$ and $F_{p}^{2 d+1-i}(d-n)$, respectively.

2. If $n, u \geq 0, i \neq 2 u+1$, and $2 d+2-i \neq 2 n+1$, then the Tate module of $C^{2 d+2-i}(n)$ is Pontrjagin dual to $C^{i}(u) / F^{i}(u)$.

3. $U^{i}(n) \cong \operatorname{Hom}_{W(k)}\left(U^{2 d+2-i}(d-n), C W(k)\right)$, for $C W(k)$ the co-Witt vectors.

From the Rost-Voevodsky theorem it follows that the motivic cohomology groups $H_{\mathcal{M}}^{i}(X, \mathbb{Z}(n))$ satisfy the conclusion of the previous theorem for $i \leq$ $n+1$ and for $n \geq \operatorname{dim} X$ as well. We mention some results and examples on $H_{\mathrm{et}}^{2 n}(X, \mathbb{Z}(n))$ as well.

If the base field is finite, then a conjecture of Lichtenbaum gives a precise conjectural description of the structure of étale motivic cohomology [20], and the conjecture is equivalent to other deep and well-known conjectures. Over local fields, our main result is:

Theorem 1.3. Let $k$ be a p-adic field of residue characteristic $p$. Then $H_{\mathrm{et}}^{i}(X, \mathbb{Z}(n))$ is the direct sum of a finite group and a group which is uniquely l-divisible for all $l \neq p$, if either $X$ has good reduction and $i \notin\{2 n-1,2 n, 2 n+1,2 n+2\}$, or if $i \notin\{n, \ldots, n+d+2\}$.

We also give a list of examples showing that the bounds are sharp, and ask some more precise questions on the structure of the groups.

Finally, let $C$ be spectrum of the ring of integers of a number field or a smooth and proper curve over a finite field. In analogy to the situation over finite fields, Lichtenbaum conjectures that if $X$ is regular, and proper over $C$, then the groups $H_{\mathrm{et}}^{i}(X, \mathbb{Z}(n))$ are finitely generated for $i \leq 2 n$, finite for $i=2 n+1$ and of cofinite type for $i \geq 2 n+2$. If $B=C-S$ is the complement of a finite, non-empty set $S$ of places, and $X$ is smooth and proper over $B$, then we expect $H_{\text {et }}^{i}(X, \mathbb{Z}(n))$ to be the direct sum of a finitely generated group and a group of cofinite type, and we raise the question if the group

$$
\operatorname{Sh}^{i, n}(X)=\operatorname{ker} H_{\mathrm{et}}^{i}(X, \mathbb{Z}(n)) \rightarrow \bigoplus_{v \in S} H^{i}\left(X \times_{B} K_{v}, \mathbb{Z}(n)\right),
$$

where $K_{v}$ is the completion of the function field of $B$ at the place $v$, is finite if $S$ contains at least one finite place. We show that the answer is (trivially) affirmative for some small values of $i$ and $n$, and prove

Theorem 1.4. If $f: X \rightarrow B$ is smooth and proper, then $S h^{3,1}(X)$ is finite if and only if the Tate-Shafarevich group $S h\left(\mathrm{Pic}_{X_{\eta} / \eta}^{0, r e d}\right)$ of the Picard variety of the generic fiber is finite. 
Notation: Throughout the paper, $n$ will be a non-negative integer. We denote $\mathbb{Z}(n)$ Bloch's motivic complex of cycles of codimension $n$, a complex of étale sheaves on the category $S m / B$ of smooth schemes over $B$, [13, Thm.1.17], by $\mathbb{Z}(n)$.

For an abelian group $A$ we denote by ${ }_{m} A$ its $m$-torsion, by $A\{l\}=\operatorname{colim}_{l^{r} l^{r}} A$ its subgroup of $l$-power torsion elements, by $A^{*}$ its Pontrjagin dual $\operatorname{Hom}(A, \mathbb{Q} / \mathbb{Z})$, by $A^{\wedge}=\lim A / m$ its completion, by $A^{\wedge l}=\lim A / l^{r}$ its $l$-adic completion, by $T A=\lim _{m} A$ its Tate module, and by $T_{l} A=\lim _{r} l^{r} A$ its $l$-adic Tate module. The subgroup of $l$-divisible elements is denoted by $l$-div $A$ and the largest $l$-divisible subgroup by $l$ - Div $A$.

We will call an $l$-power torsion group of cofinite type, if it is of the form $\left(\mathbb{Q}_{l} / \mathbb{Z}_{l}\right)^{r} \oplus F$ for a finite group $F$, and we call a torsion group of cofinite type if it is of the form $\mathbb{Q} / \mathbb{Z}\left[\frac{1}{p}\right]^{r} \oplus \mathbb{Q}_{p} / \mathbb{Z}_{p}^{s}$ (where $p$ is the characteristic of the base field in case of algebraically closed and finite fields, and the residue characteristic on case of local fields).

\section{Algebraically closed base fields}

Assume that $k$ is an algebraically closed field of characteristic $p \geq 0$.

Proposition 2.1. If $l \neq p$ is a prime number, then the l-adic cohomology groups $H_{\mathrm{et}}^{i}\left(X, \mathbb{Z}_{l}(n)\right)$ are finitely generated $\mathbb{Z}_{l}$-modules, of rank independent of $l \neq p$, and torsion free for almost all $l$. The groups $H_{\mathrm{et}}^{i}\left(X, \mathbb{Q}_{l} / \mathbb{Z}_{l}(n)\right)$ and $H_{\mathrm{et}}^{i}(X, \mathbb{Z}(n))\{l\}$ are of cofinite type, of corank independent of $l$, and cofree for almost all $l$.

Proof. By SGA 4.5, the groups $H_{\mathrm{et}}^{i}\left(X, \mathbb{Z} / l^{r}(n)\right)$ are finite, hence $H_{\mathrm{et}}^{i}\left(X, \mathbb{Z}_{l}(n)\right)=$ $\lim H_{\mathrm{et}}^{i}\left(X, \mathbb{Z} / l^{r}(n)\right)$ is a compact $\mathbb{Z}_{l}$-module, and hence finitely generated. By Gabber [5], its $l$-torsion vanishes for almost all $l$. The rank does not depend on $l$ by comparing with the Betti-number in characteristic 0 , and by the Weilconjectures in characteristic $p$. The statements for $\mathbb{Q}_{l} / \mathbb{Z}_{l}$-coefficients follows by taking the colimit, and the statement about torsion follows from the surjection $H_{\mathrm{et}}^{i}\left(X, \mathbb{Q}_{l} / \mathbb{Z}_{l}(n)\right) \rightarrow H_{\mathrm{et}}^{i+1}(X, \mathbb{Z}(n))\{l\}$.

For the $p$-part, we have the following:

Proposition 2.2. The groups $H_{\mathrm{et}}^{i}\left(X, \mathbb{Z}_{p}(n)\right)$ are the direct sum of a finitely generated free $\mathbb{Z}_{p}$-module and an extensions of a finite group by a finitely generated torsion $W(k)$-module. The groups $H_{\mathrm{et}}^{i}\left(X, \mathbb{Q}_{p} / \mathbb{Z}_{p}(n)\right)$ and $H_{\mathrm{et}}^{i}(X, \mathbb{Z}(n))\{p\}$ are the direct sum of a group of the form $\left(\mathbb{Q}_{p} / \mathbb{Z}_{p}\right)^{r}$, and an extension of a finite group by a finitely generated torsion $W(k)$-module.

Proof. We have $H_{\mathrm{et}}^{i}\left(X, \mathbb{Z} / p^{r}(n)\right) \cong H_{\mathrm{et}}^{i-n}\left(X, \nu_{r}^{n}\right)$ by [11]. By Milne [22, Lemma 1.8], the sheaf associated to the presheaf $H^{j}\left(X \times-, \nu_{r}^{n}\right)$ on the category $P f / k$ of perfect schemes over $k$ with the étale topology is represented by a commutative 
perfect group scheme over $k$, corresponding to an extension of an étale group scheme by a unipotent commutative quasi-algebraic group, whose limit is finite dimensional by loc.cit. Prop. 3.1. Taking global sections over $k$, and then the limit, the results follows. The statement about torsion coefficients follows by taking the colimit, and the statement on torsion follows from the short exact sequence

$$
0 \rightarrow H_{\mathrm{et}}^{i}(X, \mathbb{Z}(n)) \otimes \mathbb{Q}_{p} / \mathbb{Z}_{p} \rightarrow H_{\mathrm{et}}^{i}\left(X, \mathbb{Q}_{p} / \mathbb{Z}_{p}(n)\right) \rightarrow H_{\mathrm{et}}^{i+1}(X, \mathbb{Z}(n))\{p\} \rightarrow 0
$$

Indeed, since the left hand group is divisible, the cotorsion of the two groups on the right is isomorphic.

Corollary 2.3. The torsion subgroup $\operatorname{Tor} H_{\mathrm{et}}^{i}(X, \mathbb{Z}(n))$ is a direct summand of $H_{\text {et }}^{i}(X, \mathbb{Z}(n))$.

Proof. The proposition shows that Tor $H_{\mathrm{et}}^{i}(X, \mathbb{Z}(n))$ is the direct sum of a divisible group and a group of finite exponent. The result now follows from [15, Thm. 16].

Proposition 2.4. Let $F / k$ be an extension of algebraically closed fields. Then for $l \neq p$, the base change maps

$$
\begin{gathered}
H_{\mathrm{et}}^{i}(X, \mathbb{Z}(n))\{l\} \stackrel{\sim}{\rightarrow} H_{\mathrm{et}}^{i}\left(X_{F}, \mathbb{Z}(n)\right)\{l\} \\
H_{\mathrm{et}}^{i}(X, \mathbb{Z}(n))^{\wedge l} \stackrel{\sim}{\rightarrow} H_{\mathrm{et}}^{i}\left(X_{F}, \mathbb{Z}(n)\right)^{\wedge l}
\end{gathered}
$$

are isomorphisms. For $l=p$, then are injective with cokernel of bounded exponent. Furthermore we have

$$
H_{\mathrm{et}}^{i}(X, \mathbb{Z}(n)) \otimes \mathbb{Q} / \mathbb{Z} \cong H_{\mathrm{et}}^{i}\left(X_{F}, \mathbb{Z}(n) \otimes \mathbb{Q} / \mathbb{Z}\right.
$$

Proof. Consider the map of short exact coefficient sequences:

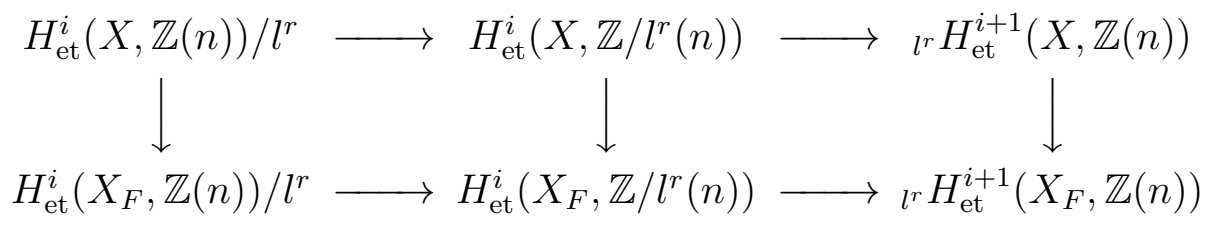

Since the middle map is an isomorphism by the smooth and proper base change theorem for $l \neq p$, and is injective with a cokernel bounded independently of $r$ for $l=p$ because $H_{\mathrm{et}}^{i}\left(X, \mathbb{Z} / p^{r}(n)\right) \cong H_{\mathrm{et}}^{i-n}\left(X, \nu_{r}^{n}\right)$ and [22, Lemma 1.8], it suffices to show that the outer maps are injective. We write $F$ as a colimit of finitely generated $k$-algebras $A$, and note that $k \subseteq A$ is split because $k$ is algebraically closed. Since étale cohomology commutes with limits with affine transition maps, the result follows. 
Note that no information can be deduced about a group from knowing its completion and torsion subgroup, even in the absence of uniquely divisible groups. For example, $G=\mathbb{Z}$ and $G=\oplus_{l} \mathbb{Z}_{(l)}$ both satisfy $G^{\wedge l} \cong \mathbb{Z}_{l}$ and $G \otimes \mathbb{Q}_{l} / \mathbb{Z}_{l} \cong \mathbb{Q}_{l} / \mathbb{Z}_{l}$ for all $l$.

Proof. (Theorem 1.1). It suffices to show that $H_{\text {et }}^{i}(X, \mathbb{Z}(n)) \otimes \mathbb{Q} / \mathbb{Z}=0$. Indeed, then the short exact sequence

$$
0 \rightarrow \operatorname{Tor} H_{\text {et }}^{i}(X, \mathbb{Z}(n)) \rightarrow H_{\text {et }}^{i}(X, \mathbb{Z}(n)) \rightarrow H_{\text {et }}^{i}(X, \mathbb{Z}(n)) \otimes \mathbb{Q} \rightarrow 0
$$

shows that $H_{\mathrm{et}}^{i}(X, \mathbb{Z}(n))$ modulo its torsion subgroup is uniquely divisible, and the structure of the torsion subgroup is given in Propositions 2.1 and 2.2 .

To show $H_{\text {et }}^{i}(X, \mathbb{Z}(n)) \otimes \mathbb{Q} / \mathbb{Z}=0$, we take a (normal, reduced) scheme $S$ of finite type over $\mathbb{Z}$ and a scheme $\mathcal{X}$ over $S$ such that $X$ is the base change to $k$ of the generic fiber $\mathcal{X}_{\eta}$. From Proposition 2.4 we know that $H_{\text {et }}^{i}\left(\mathcal{X}_{\bar{\eta}}, \mathbb{Z}(n)\right) \otimes \mathbb{Q} / \mathbb{Z} \cong$ $H_{\text {et }}^{i}(X, \mathbb{Z}(n)) \otimes \mathbb{Q} / \mathbb{Z}$, hence it suffices to consider the case $k=\bar{\eta}$. Let $N$ be $H_{\text {et }}^{i}(X, \mathbb{Z}(n))$ modulo its torsion subgroup, it suffices to show that $N$ is divisible. From the exact sequence $0 \rightarrow l$ - $\operatorname{div} N \rightarrow N \rightarrow N^{\wedge l}$ we see that it is enough to show that for all $l$ the map

$$
N \rightarrow N^{\wedge l} \subseteq H_{\mathrm{et}}^{i}\left(X, \mathbb{Z}_{l}(n)\right) / \text { Tor } \subseteq H_{\mathrm{et}}^{i}\left(X, \mathbb{Q}_{l}(n)\right)
$$

is the zero map. Since the smooth locus of $\mathcal{X}$ is open (and non-empty because $X$ is smooth), we can find a point Spec $\mathbb{F}$ of $S$ with finite residue field such that the fiber $\mathcal{X}_{\mathbb{F}}$ is smooth. If $l \neq p$, we have an isomorphism $H_{\mathrm{et}}^{i}\left(X_{\overline{\mathbb{F}}}, \mathbb{Q}_{l}(n)\right) \cong H_{\mathrm{et}}^{i}\left(X_{\bar{\eta}}, \mathbb{Q}_{l}(n)\right)$, compatible with the action of $\operatorname{Gal}(\eta) \rightarrow \operatorname{Gal}(\mathbb{F})$, by the smooth and proper base change theorem. For $l=p$, we can assume that $S$ is of finite type over a finite field contained in $\mathbb{F}$, and the same statement holds by Gros-Suwa [12, Thm. II 2.1]. Now since

$$
N \subseteq H_{\text {et }}^{i}(X, \mathbb{Q}(n)) \cong H_{\mathcal{M}}^{i}(X, \mathbb{Q}(n))=\underset{L \supseteq \eta \text { folinite }}{\operatorname{col}} H_{\mathcal{M}}^{i}\left(X_{L}, \mathbb{Q}(n)\right),
$$

every element of $N$ has finite orbit under $\operatorname{Gal}(\eta)$. In particular, the arithmetic Frobenius $\varphi$ has finite order acting on any element of $N$. On the other hand, by the Weil conjectures and its $p$-adic version, the eigenvalues of $\varphi$ acting on $H_{\text {et }}^{i}\left(X_{\overline{\mathbb{F}}}, \mathbb{Q}_{l}(n)\right)$ have absolute value $p^{n-\frac{i}{2}}$, hence there is no element of finite order.

Proof. (Proposition 1.2). Since $T_{l} H_{\mathrm{et}}^{i+1}(X, \mathbb{Z}(n))$ is torsion free, we get from the coefficient sequence that

$$
F^{i}(n) \oplus P_{c}^{i}(n) \cong \operatorname{Tor} H_{\mathrm{et}}^{i}(X, \mathbb{Z}(n))^{\wedge} \cong \operatorname{Tor} \prod_{l} H_{\mathrm{et}}^{i}\left(X, \mathbb{Z}_{l}(n)\right)
$$


If $l \neq p$, then $F^{i}(n)$ does not depend on $n$ because $H_{\text {et }}^{i}\left(X, \mathbb{Z}_{l}\right) \cong H_{\text {et }}^{i}\left(X, \mathbb{Z}_{l}(n)\right)$ for every $n$. Taking the inverse limit of Poincaré-duality we obtain

$$
H_{\mathrm{et}}^{i}\left(X, \mathbb{Z}_{l}(n)\right) \cong H_{\mathrm{et}}^{2 d-i}\left(X, \mathbb{Q}_{l} / \mathbb{Z}_{l}(d-n)\right)^{*} .
$$

Since $\operatorname{Hom}(D, \mathbb{Q} / \mathbb{Z})$ is uniquely divisible for a divisible group $D$, the map

$$
H_{\mathrm{et}}^{2 d-i+1}\left(X, \mathbb{Z}_{l}(d-n)\right)^{*} \rightarrow H_{\mathrm{et}}^{2 d-i}\left(X, \mathbb{Q}_{l} / \mathbb{Z}_{l}(d-n)\right)^{*}
$$

induces an isomorphism

$$
\left(\operatorname{Tor} H_{\mathrm{et}}^{2 d-i+1}\left(X, \mathbb{Z}_{l}(d-n)\right)\right)^{*} \cong\left(\operatorname{Cotor} H_{\mathrm{et}}^{2 d-i}\left(X, \mathbb{Q}_{l} / \mathbb{Z}_{l}(d-n)\right)\right)^{*} .
$$

For the $p$-part, we use Milne's duality [22, Thm.1.11], see also [12, Cor. 3.26]. Let $\underline{U}^{i}\left(X, \nu_{r}(n)\right)$ be the unipotent part and $\underline{D}^{i}\left(X, \nu_{r}(n)\right)$ be the étale quotient of the finite dimensional pro-group scheme $\underline{H}^{i-n}\left(X \times-, \nu_{r}(n)\right)$ on $P f / k$, see also [12, Cor. 3.25]. Taking $k$-rational points we obtain $H_{\text {et }}^{i}\left(X, \mathbb{Z} / p^{r}(n)\right)$, and since $\underline{U}^{i}(X, \nu .(n))$ is finite dimensional by loc.cit. Prop. 3.1, we get $U^{i}(n) \cong$ $\operatorname{colim}_{r} \underline{U}^{i-n-1}\left(X, \nu_{r}(n)\right)(k)$, and $F_{p}^{i}(n)$ is isomorphic both to the cotorsion of $\operatorname{colim}_{r} \underline{D}^{i-n-1}\left(X, \nu_{r}(n)\right)(k)$ and to the torsion in $\lim _{r} \underline{D}^{i-n}\left(X, \nu_{r}(n)\right)(k)$. Hence $D^{i}(n) \cong \operatorname{Hom}\left(D^{2 d+1-i}(d-n), \mathbb{Q}_{p} / \mathbb{Z}_{p}\right)$, which gives duality for $F_{p}^{i}(n)$ exactly as above, and $U^{i}(n) \cong \operatorname{Hom}_{W(k)}\left(U^{2 d+2-i}(d-n), C W(k)\right)$.

\section{Remarks}

1. The method of Colliot-Thélène and Raskind [2, Th. 1.8, 2.2] yields similar results away from the characteristic $p$.

2. It would be interesting to write down the duality pairing between $U^{i}(n)$ and $U^{2 d+2-i}(d-n)$ in terms of the motivic cohomology groups directly.

3. The Beilinson-Soulé vanishing conjecture is equivalent to the vanishing of $D^{i}(n)=0$ for $i<0$.

4. If the base field has characteristic $p>0$ and if we assume Parshin's conjecture, then $D^{i}(n)=0$ for $i<n[6]$. A more careful analysis shows that if the base-field has transcendence degree $r$ over the finite prime field, then $H_{\mathrm{et}}^{i}(X, \mathbb{Q}(n))=0$ for $i<\max \{n, 2 n-r\}$ under Parshin's conjecture.

5. There is no non-degenerate pairing between $D^{i}(n)$ and some $D^{j}(d-n)$, because for the algebraic closure of the rationals, $D^{1}(n)$ is infinite dimensional for every $n>0$. 


\section{Examples}

1. We have $F^{0}(n)=F^{1}(n)=F^{2 d}(n)=0, F^{2}(n)$ is the prime to $p$-torsion of the Neron-Severi group, and $F^{3}(n)$ is the prime to $p$-cotorsion of the Brauer group.

2. If $X$ is a supersingular abelian surface or $\mathrm{K} 3$ surface, then $H_{\mathrm{et}}^{3}(X, \mathbb{Z}(1))$ has a direct summand isomorphic to $k$, dual to itself.

3. We have

$$
H_{\mathrm{et}}^{1}(\mathbb{Q}, \mathbb{Z}(1)) \cong \mathbb{Q}^{\times} \cong \mathbb{Z} / 2 \oplus \bigoplus_{p} \mathbb{Z}
$$

is the Galois invariants of

$$
H_{\mathrm{et}}^{1}(\overline{\mathbb{Q}}, \mathbb{Z}(1)) \cong \overline{\mathbb{Q}}^{\times} \cong \mathbb{Q} / \mathbb{Z} \oplus \bigoplus_{\mathfrak{p}} \mathbb{Q}
$$

where $\mathfrak{p}$ runs over all finite places of $\overline{\mathbb{Q}}$. This can be explained by the Galois cohomology sequence associated to the short exact sequence of Galois modules

$$
0 \rightarrow \operatorname{Tor} H_{\mathrm{et}}^{i}(\bar{X}, \mathbb{Z}(n)) \rightarrow H_{\mathrm{et}}^{i}(\bar{X}, \mathbb{Z}(n)) \rightarrow D^{i}(n) \rightarrow 0 .
$$

In particular, the action of the Galois group is not compatible with the decomposition in Theorem 1.1.

\section{The étale Chow-group}

This section gives an overview over some known results in degree $2 n$.

\section{Equivalence relations}

Let $X$ be again a smooth and projective scheme over an algebraically closed field. The intersection and cup product gives us a diagram of pairings

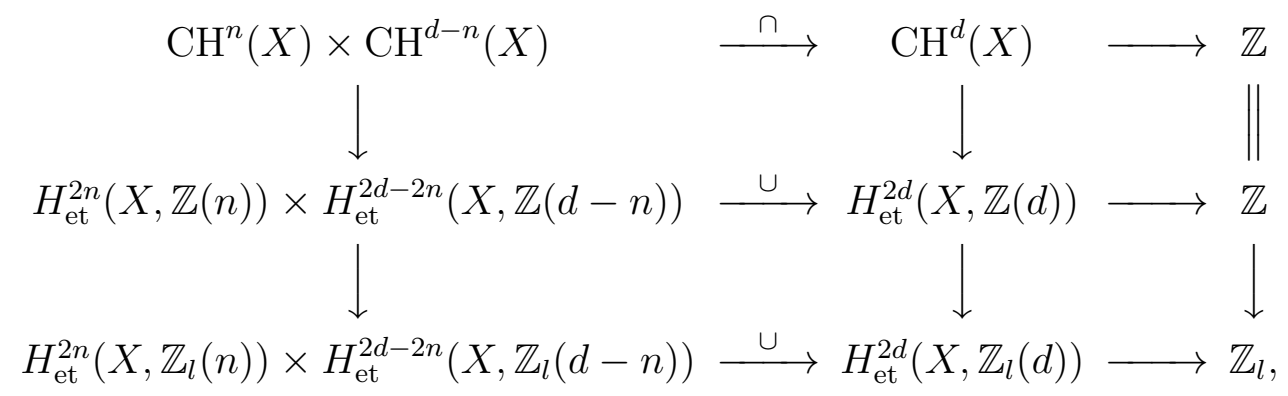

and the kernels of these pairings are the subgroups of elements numerically equivalent to zero, $\mathrm{CH}_{\text {num }}^{n}(X) \subseteq \mathrm{CH}^{n}(X)$ and $H_{\text {num }}^{2 n}(X, \mathbb{Z}(n)) \subseteq H_{\text {et }}^{2 n}(X, \mathbb{Z}(n))$. The lower pairing is non-degenerate modulo torsion. 
Proposition 3.1. The map

$$
\mathrm{CH}^{n}(X) / \mathrm{CH}_{n u m}^{n}(X) \rightarrow H_{\mathrm{et}}^{2 n}(X, \mathbb{Z}(n)) / H_{n u m}^{2 n}(X, \mathbb{Z}(n))
$$

is an isomorphism of finitely generated free abelian groups.

Proof. We write $A^{D}=\operatorname{Hom}(A, \mathbb{Z})$ for an abelian group $A$. We have nondegenerate pairings on $N=\mathrm{CH}^{n}(X) / \mathrm{CH}_{\text {num }}^{n}(X)$ to $\mathbb{Z}$, which shows that these groups are torsion free. On the other hand, since the pairings above are compatible, and the lower pairing is perfect after tensoring with $\mathbb{Q}_{l}, N \otimes \mathbb{Q}_{l}$ injects into the finite dimensional $\mathbb{Q}_{l}$-vector space $H_{\mathrm{et}}^{2 n}\left(X, \mathbb{Q}_{l}(n)\right)$. This implies that $N \otimes \mathbb{Q}$ is finite dimensional. Let $\left\{n_{1}, \ldots, n_{r}\right\}$ be elements of $N$ which form a basis of $N \otimes \mathbb{Q}$, and $N_{1} \subseteq N$ be the submodule generated by $\left\{n_{1}, \ldots, n_{r}\right\}$. Since $N / N_{1}$ is torsion, we have $\left(N / N_{1}\right)^{D}=0$, hence the map

$$
M=\mathrm{CH}^{d-n}(X) / \mathrm{CH}_{\text {num }}^{d-n}(X) \subseteq N^{D} \rightarrow N_{1}^{D}
$$

is injective. Thus $M$ injects into a finitely generated free abelian group, hence is finitely generated free. The same argument applies to $H_{\mathrm{et}}^{2 n}(X, \mathbb{Z}(n)) / H_{\text {num }}^{2 n}(X, \mathbb{Z}(n))$. To get the isomorphism, consider the commutative diagram

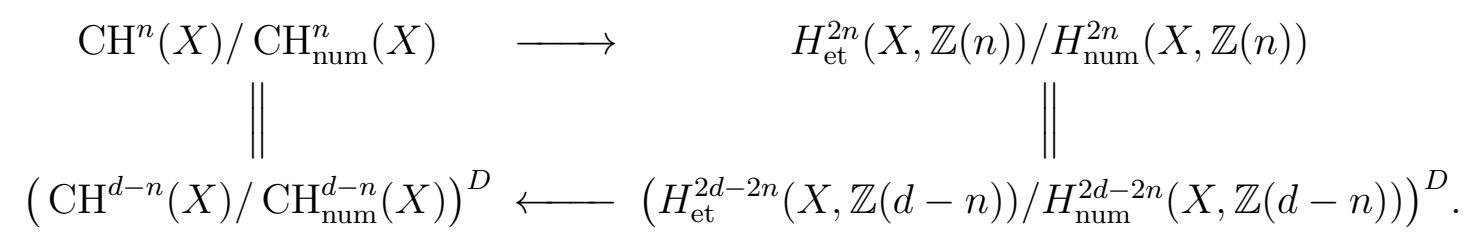

Since $\mathrm{CH}^{n}(X)_{\mathbb{Q}} \cong H_{\mathrm{et}}^{2 n}(X, \mathbb{Q}(n))$, the upper map is rationally surjective, hence all groups are finitely generated free abelian groups of the same rank, and it follows that all maps are isomorphisms.

We define the groups $\mathrm{CH}_{\text {hom }}^{n}(X)$ and $H_{\text {hom }}^{2 n}(X, \mathbb{Z}(n))$ of cycles homologically equivalent to zero to be the kernel of the composition, and of the second map in

$$
\mathrm{CH}^{n}(X) \rightarrow H_{\mathrm{et}}^{2 n}(X, \mathbb{Z}(n)) \rightarrow \prod_{l} H_{\mathrm{et}}^{2 n}\left(X, \mathbb{Z}_{l}(n)\right),
$$

respectively. Since the second map factors through the subgroup $\lim _{m} H_{\mathrm{et}}^{2 n}(X, \mathbb{Z}(n)) / m$, $H_{\text {hom }}^{2 n}(X, \mathbb{Z}(n))$ is the group of divisible elements.

Lemma 3.2. The group $H_{h o m}^{2 n}(X, \mathbb{Z}(n))$ is the maximal divisible subgroup of $H_{\mathrm{et}}^{2 n}(X, \mathbb{Z}(n))$, and the map

$$
\mathrm{CH}^{n}(X) / \mathrm{CH}_{h o m}^{n}(X) \rightarrow H_{\mathrm{et}}^{2 n}(X, \mathbb{Z}(n)) / H_{h o m}^{2 n}(X, \mathbb{Z}(n))
$$

is injective with torsion cokernel. 
Proof. If an element in $\mathrm{CH}^{n}(X)$ maps to $H_{\text {hom }}^{2 n}(X, \mathbb{Z}(n))$, then it vanishes in $l$-adic cohomology, hence is contained in $\mathrm{CH}_{\text {hom }}^{n}(X)$ which implies injectivity. The cokernel is torsion because the Chow group and étale Chow group agree rationally.

It is conjectured that $\mathrm{CH}_{\text {hom }}^{n}(X) \subseteq \mathrm{CH}_{\text {num }}^{n}(X)$ agree up to a torsion group. The same argument as in the Lemma gives:

Lemma 3.3. The map

$$
\mathrm{CH}_{\text {num }}^{n}(X) / \mathrm{CH}_{\text {hom }}^{n}(X) \rightarrow H_{\text {num }}^{2 n}(X, \mathbb{Z}(n)) / H_{\text {hom }}^{2 n}(X, \mathbb{Z}(n))
$$

is injective with torsion cokernel.

A cycle is algebraically equivalent to zero if it lies in the image of some map

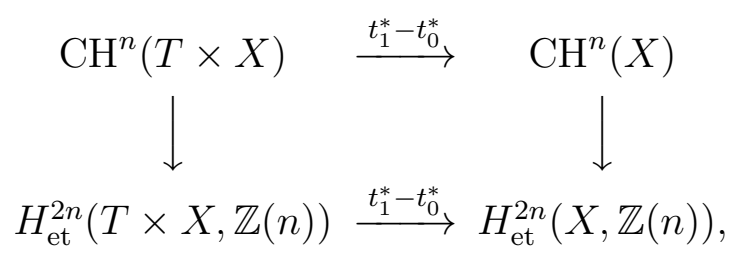

where $T$ is a smooth connected scheme $T$ (which we can assume to be a smooth curve) with closed points $t_{0}, t_{1} \in T$.

We obtain compatible filtrations on Chow groups and étale Chow groups

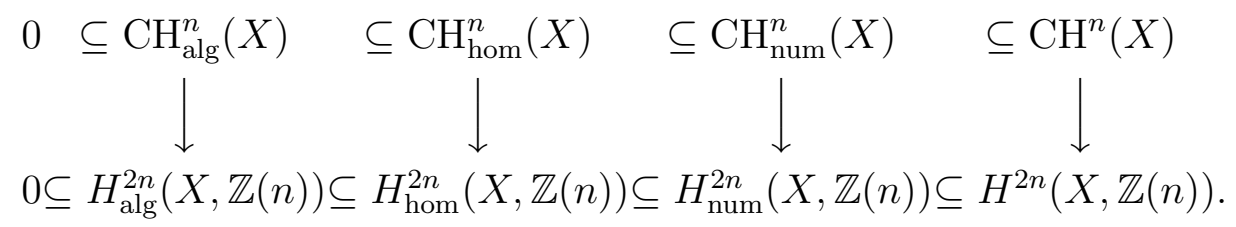

The upper and lower groups agree rationally.

\section{Examples}

1. (Bloch and Esnault [1) There are 3-dimensional complete intersections $X$ over the algebraic closure of a number field such that $\mathrm{CH}_{\text {hom }}^{2}(X)$ is not $l$-divisible, and $C H^{2}(X)\{l\}=0$ for some primes $l$.

2. (Schoen [32]) The triple self-product of an elliptic curve over an algebraically closed field of characteristic 0 can have infinite $\mathrm{CH}^{n}(X) / l$ for $2 \leq n \leq d-1$, in particular $\mathrm{CH}_{\text {hom }}^{n}(X) / l$ is infinite. From the injectivity of $\mathrm{CH}_{\text {hom }}^{2}(X) \subseteq$ $\mathrm{CH}^{2}(X) \rightarrow H_{\mathrm{et}}^{4}(X, \mathbb{Z}(2))$ and finiteness of $H_{\mathrm{et}}^{4}(X, \mathbb{Z} / l(2))$ it follows that there is a subgroup of infinite rank in $\mathrm{CH}^{2}(X)$ which becomes divisible in $H_{\mathrm{et}}^{4}(X, \mathbb{Z}(2))$. 
3. (Schoen [30]) Over the algebraic closure of a finite field of characteristic larger than 2, and assuming Tate's conjecture and semi-simplicity of the Frobenius action, the map $\mathrm{CH}_{\text {hom }}^{n}(X) \rightarrow H_{\mathrm{et}}^{2 n-1}\left(X, \mathbb{Q}_{l} / \mathbb{Z}_{l}(n)\right)$ is surjective for almost all $l \neq p$ and $n$ if the dimension of $X$ is at most 4 , and in general under some additional hypothesis. Note that over the algebraic closure of a finite field, $\mathrm{CH}_{\text {hom }}^{n}(X)$ is conjectured to be a torsion group.

4. (Schoen [29]) For any $n \geq 2$ there are varieties of dimension $2 n-1$ such that the Griffiths group $G r^{n}(X)=\mathrm{CH}_{\text {hom }}^{n}(X) / \mathrm{CH}_{\text {alg }}^{n}(X)$ has torsion elements.

5. (Schoen [31]) There are smooth, projective varieties $V$, such that the corank of $\mathrm{CH}^{r}(X)\{l\}$ is infinite for all $r$ in the range $2<r<\operatorname{dim}(X)$.

\section{Representability}

Recall that a group homomorphism from $\mathrm{CH}_{\text {alg }}^{n}(X)$ to the $k$-rational points of an abelian variety $A$ is regular, if for every pointed smooth connected variety $t_{0} \in T$ and correspondence $\Gamma \in \mathrm{CH}^{n}(T \times X)$, the composition with the map

$$
T(k) \rightarrow \mathrm{CH}_{\mathrm{alg}}^{n}(X), \quad t \mapsto t^{*} \Gamma-t_{0}^{*} \Gamma
$$

is the map induced on closed points by a morphism of schemes $T \rightarrow A$. We use the same concept for the étale Chow group:

Definition 3.4. A homomorphism from $H_{\text {alg }}^{2 n}(X, \mathbb{Z}(n))$ to the k-rational points of an abelian variety $A$ is regular, if for every pointed smooth connected variety $t_{0} \in T$ and element $\Gamma \in H_{\text {alg }}^{2 n}(T \times X, \mathbb{Z}(n))$, the composition with

$$
T(k) \rightarrow H_{a l g}^{2 n}(X, \mathbb{Z}(n)), \quad t \mapsto t^{*} \Gamma-t_{0}^{*} \Gamma
$$

is the map induced on closed points by a morphism of varieties $T \rightarrow A$.

Theorem 3.5. There is a universal object $\rho_{n}: H_{\text {alg }}^{2 n}(X, \mathbb{Z}(n)) \rightarrow A_{n}$ for regular homomorphisms from $H_{\text {alg }}^{2 n}(X, \mathbb{Z}(n))$ to abelian varieties.

Proof. This follows by the argument of Serre [33] and H. Saito [27] because the dimension of surjective maps to abelian varieties is bounded.

In [24], 25], Murre studied the situation for Chow groups, and he proved that a universal homomorphism to abelian varieties exists for dimension 0 , and codimensions 1 and 2, so also [17]. 


\section{Other bases}

\section{Finite fields}

We mention a conjecture on the structure of motivic cohomology groups over finite fields:

Conjecture 4.1. (Lichtenbaum [20])

$$
H_{\mathrm{et}}^{i}(X, \mathbb{Z}(n))= \begin{cases}\text { finite } & i \neq 2 n, 2 n+2, \\ \text { finitely generated } & i=2 n, \\ \text { cofinite type } & i=2 n+2 .\end{cases}
$$

We note that a small modification of étale motivic cohomology, called Weilétale cohomology, yields groups which are conjecturally finitely generated for all $i,[21,8]$.

Example 4.2. For $X$ a point we have

$$
H_{\mathrm{et}}^{i}\left(\mathbb{F}_{q}, \mathbb{Z}(n)\right)= \begin{cases}\mathbb{Z} & (i, n)=(0,0) \\ \mathbb{Q} / \mathbb{Z} & (i, n)=(2,0) \\ \mathbb{Z} / q^{n}-1 & i=1, n>0 \\ 0 & \text { otherwise }\end{cases}
$$

For $n=0$ this is the calculation of Galois cohomology, and for $n \geq 1$, the groups $H_{\mathrm{et}}^{i}\left(\mathbb{F}_{q}, \mathbb{Z}(n)\right)$ are annihilated by $q^{n}-1$ by [9, Thm. 4.6], hence they are isomorphic to the well-known groups $H_{\mathrm{et}}^{i-1}\left(\mathbb{F}_{q}, \mathbb{Q} / \mathbb{Z}(n)\right)$.

Proposition 4.3. Lichtenbaum's conjecture is true for curves.

Proof. By Soulé [35], $H_{\text {et }}^{i}(X, \mathbb{Z}(n))$ is torsion unless $(i, n)=(0,0)$ or $(2,1)$, in which case the groups are $\mathbb{Z}$ and $\mathbb{Z} \oplus$ (finite), respectively. On the other hand, $H_{\mathrm{et}}^{i}(X, \mathbb{Q} / \mathbb{Z}(n))$ is of cofinite type, and finite unless $(i, n)=(0,0),(1,0),(2,1)$, or $(3,1)$. It follows that $\operatorname{Tor} H_{\mathrm{et}}^{i}(X, \mathbb{Z}(n))$ is finite unless $(i, n)=(1,0),(2,0),(3,1)$, or $(4,1)$. Finally, $H_{\text {et }}^{1}(X, \mathbb{Z}(0))=0$ and finiteness of $H_{\text {et }}^{3}(X, \mathbb{Z}(1))=\operatorname{Br}(X)$, follows from Tate's theorem.

Proposition 4.4. For $i<2 n$, Conjecture 4.1 is true up to a uniquely divisible group, and true if and only Parshin's conjecture holds. For $i \geq 2 n+2$, it holds unconditionally, and for $i=2 n+1$, it is equivalent to Tate's conjecture on the surjectivity of the cycle map.

The conjecture holds for all $X$ and $n$ in all degrees if and only if Tate's conjecture holds, the Frobenius acts semi-simply at the eigenvalue 1 on $H_{\mathrm{et}}^{2 n}\left(\bar{X}, \mathbb{Q}_{l}(n)\right)$, and rational and numerical equivalence agree up to torsion on codimension $n$ cycles, for all $X$ and $n$. 
Proof. By the Weil conjectures and Gabber's theorem, the groups $H_{\mathrm{et}}^{i}(X, \mathbb{Q} / \mathbb{Z}(n))$ and $\prod_{l} H_{\mathrm{et}}^{i}\left(X, \mathbb{Z}_{l}(n)\right)$ are finite unless $i=2 n, 2 n+1$. Thus the conjecture follows for $i \geq 2 n+2$, because $H_{\mathrm{et}}^{i-1}(X, \mathbb{Q} / \mathbb{Z}(n))$ surjects onto the torsion group $H_{\mathrm{et}}^{i}(X, \mathbb{Z}(n))$, which implies that they are of cofinite type, and finite for $i>2 n+2$.

For $i<2 n$, the finite group $H_{\mathrm{et}}^{i-1}(X, \mathbb{Q} / \mathbb{Z}(n))$ surjects onto Tor $H_{\mathrm{et}}^{i}(X, \mathbb{Z}(n))$, and since $H_{\mathrm{et}}^{i}(X, \mathbb{Z}(n)) \otimes \mathbb{Q} / \mathbb{Z}=0$, the short exact sequence

$$
0 \rightarrow \operatorname{Tor} H_{\mathrm{et}}^{i}(X, \mathbb{Z}(n)) \rightarrow H_{\mathrm{et}}^{i}(X, \mathbb{Z}(n)) \rightarrow H_{\mathrm{et}}^{i}(X, \mathbb{Q}(n)) \rightarrow 0
$$

shows that $H_{\mathrm{et}}^{i}(X, \mathbb{Z}(n))$ modulo torsion is uniquely divisible. The vanishing of this uniquely divisible subgroup is a restatement of Parshin's conjecture.

For $i=2 n$ consider the sequence

$$
0 \rightarrow H_{\mathrm{et}}^{2 n}(X, \mathbb{Z}(n))^{\wedge l} \rightarrow H_{\mathrm{et}}^{2 n}\left(X, \mathbb{Z}_{l}(n)\right) \rightarrow T_{l} H_{\mathrm{et}}^{2 n+1}(X, \mathbb{Z}(n)) \rightarrow 0 .
$$

The group of cofinite type $H_{\mathrm{et}}^{2 n+1}(X, \mathbb{Z}(n))$ is finite if and only if its Tate module vanishes if and only if the injection $H_{\mathrm{et}}^{2 n}(X, \mathbb{Z}(n))^{\wedge l} \rightarrow H_{\mathrm{et}}^{2 n}\left(X, \mathbb{Z}_{l}(n)\right)$ of finitely generated $\mathbb{Z}_{l}$-modules is an isomorphism. This is equivalent to Tate's conjecture, because in the composition

$$
C H^{n}(X) \otimes \mathbb{Z}_{l} \rightarrow H_{\mathrm{et}}^{2 n}(X, \mathbb{Z}(n)) \otimes \mathbb{Z}_{l} \rightarrow H_{\mathrm{et}}^{2 n}(X, \mathbb{Z}(n))^{\wedge l}
$$

the first map is rationally an isomorphism, and the second map is surjective by Nakayama's Lemma.

The final statement is [8, Thm. 8.4]. Indeed, under all those conjectures, Weilétale cohomology and étale cohomology agree in degrees $\leq 2 n$ and $H_{\mathrm{et}}^{2 n+1}(X, \mathbb{Z}(n)) \cong$ Tor $H_{W}^{2 n+1}(X, \mathbb{Z}(n))$ by loc.cit. Thm. 7.1.

\section{Local fields}

Theorem 4.5. Let $k$ be a p-adic field of residue characteristic $p$. Then $H_{\mathrm{et}}^{i}(X, \mathbb{Z}(n))$ is the direct sum of a finite group and a group which is uniquely l-divisible for all $l \neq p$, if one of the following two conditions holds

1. $X$ has good reduction and $i \notin\{2 n-1,2 n, 2 n+1,2 n+2\}$

2. $i \notin\{n, \ldots, n+d+2\}$

For $i=2 n-1$ and $X$ with good reduction, or for $i=n$ in general, $\operatorname{Tor} H_{\mathrm{et}}^{i}(X, \mathbb{Z}(n))$ is finite plus p-torsion.

Proof. Assume first that $X$ has a smooth model $\mathcal{X}$ with closed fiber $Y$. Then after inverting $p$, purity [4] gives an exact sequence

$$
\cdots \rightarrow H_{\mathrm{et}}^{i}(\mathcal{X}, \mathbb{Q} / \mathbb{Z}(n)) \rightarrow H_{\mathrm{et}}^{i}(X, \mathbb{Q} / \mathbb{Z}(n)) \rightarrow H_{\mathrm{et}}^{i-1}(Y, \mathbb{Q} / \mathbb{Z}(n-1)) \rightarrow \cdots .
$$


The left hand term is isomorphic to $H_{\mathrm{et}}^{i}(Y, \mathbb{Q} / \mathbb{Z}(n))$ by the proper base change theorem. From the Weil conjectures and Gabber's theorem [5], we know that $H_{\text {et }}^{j}(Y, \mathbb{Q} / \mathbb{Z}(u))$ is finite for $j \neq 2 u, 2 u+1$, and it follows that $H_{\text {et }}^{i}(X, \mathbb{Q} / \mathbb{Z}(n))$ is finite for $i \neq 2 n-1,2 n, 2 n+1$ after inverting $p$. In particular, $H_{\text {et }}^{i}(X, \mathbb{Z}(n)) \otimes \mathbb{Q} / \mathbb{Z}$ is a $p$-group, and Tor $H_{\mathrm{et}}^{i+1}(X, \mathbb{Z}(n))$ is the direct sum of a $p$-group of cofinite type and a finite group, hence a direct summand [15]. Finally, the sequence

$$
0 \rightarrow \operatorname{Tor} H_{\mathrm{et}}^{i}(X, \mathbb{Z}(n)) \rightarrow H_{\mathrm{et}}^{i}(X, \mathbb{Z}(n)) \rightarrow H_{\mathrm{et}}^{i}(X, \mathbb{Q}(n)) \rightarrow(p-\text { group }) \rightarrow 0
$$

show that $H_{\mathrm{et}}^{i}(X, \mathbb{Z}(n))$ modulo its torsion subgroup is uniquely $l$-divisible for all $l \neq p$.

For general $X$, Kahn [14, Thm.6 a)] uses a strengthening of the above argument to show that, for any $X, H_{\mathrm{et}}^{i}(X, \mathbb{Q} / \mathbb{Z}(n))$ is finite after localizing at $l \neq p$ for $i \notin\{n, \ldots, n+d+1\}$. The rest of the proof continues as above.

Example 4.6 (Weight 0,1). The bounds in the good reduction case are sharp. If $X$ has a rational point, then for $r=\left[k: \mathbb{Q}_{p}\right]$, we obtain

$$
\begin{array}{ll}
i=2 n-1 & H_{\mathrm{et}}^{1}(X, \mathbb{Z}(1)) \cong k^{\times} \cong \mathbb{Z} \oplus \mathbb{Z}_{p}^{r} \oplus(\text { finite }) \\
i=2 n & H_{\mathrm{et}}^{0}(k, \mathbb{Z}(0)) \cong \mathbb{Z} \\
& H_{\mathrm{et}}^{2}(X, \mathbb{Z}(1)) \cong \operatorname{Pic}(X) \cong(\text { fin.gen. }) \oplus \mathbb{Z}_{p}^{r \operatorname{dim} \operatorname{Pic}_{X}^{0}} \\
i=2 n+1 & H_{\mathrm{et}}^{3}(k, \mathbb{Z}(1)) \cong \operatorname{Br}(k) \cong \mathbb{Q} / \mathbb{Z} \\
i=2 n+2 & H_{\mathrm{et}}^{2}(k, \mathbb{Z}(0)) \cong \operatorname{Hom}\left(G_{k}, \mathbb{Q} / \mathbb{Z}\right) \cong \mathbb{Q} / \mathbb{Z} \oplus\left(\mathbb{Q}_{p} / \mathbb{Z}_{p}\right)^{r} \oplus(\text { finite })
\end{array}
$$

The structure of the Picard group follows from a theorem of Mattuck. The former groups have free summands, and the latter have infinite torsion summands.

Moreover, a trace argument shows that $H_{\mathrm{et}}^{i}(k, \mathbb{Z}(n))$ is a direct summand of $H_{\mathrm{et}}^{2 d+i}(X, \mathbb{Z}(d+n))$, i.e. the above phenomena occur for higher weights as well.

Example 4.7 (Curves, weight 1). If $C$ is a curve of genus $g$ over a $p$-adic field, then

$$
\begin{aligned}
& H_{\mathrm{et}}^{1}(C, \mathbb{Z}(1)) \cong k^{\times} \cong \mathbb{Z} \oplus \mathbb{Z}_{p}^{r} \oplus(\text { finite }) \\
& H_{\mathrm{et}}^{2}(C, \mathbb{Z}(1)) \cong \operatorname{Pic} C \cong \mathbb{Z} \oplus \mathbb{Z}_{p}^{g r} \oplus(\text { finite }) \\
& H_{\mathrm{et}}^{3}(C, \mathbb{Z}(1)) \cong \operatorname{Br} C \cong \mathbb{Q} / \mathbb{Z} \oplus\left(\mathbb{Q}_{p} / \mathbb{Z}_{p}\right)^{g r} \oplus(\text { finite })
\end{aligned}
$$

The Brauer group is dual to the Picard group by Lichtenbaum [19].

Example 4.8 (Weight $d+1$ ). From the isomorphism

$$
\left(\operatorname{Tor} H_{\mathrm{et}}^{2}(X, \mathbb{Z})\right)^{*} \cong H_{\mathrm{et}}^{1}(X, \mathbb{Q} / \mathbb{Z})^{*}=\pi_{1}^{a b}(X),
$$

and Poincaré duality $H_{\mathrm{et}}^{1}(X, \mathbb{Z} / m)^{*} \cong H_{\mathrm{et}}^{2 d+1}(X, \mathbb{Z} / m(d))$, we obtain a short exact sequence

$$
0 \rightarrow H_{\mathrm{et}}^{2 d+1}(X, \mathbb{Z}(d+1))^{\wedge} \rightarrow \pi_{1}^{a b}(X) \rightarrow T H_{\mathrm{et}}^{2 d+2}(X, \mathbb{Z}(d+1)) \rightarrow 0 .
$$


If $X$ is a curve, then $H_{\mathrm{et}}^{3}(X, \mathbb{Z}(2)) \cong S K_{1}(X)$, and we recover the exact sequence of Saito [28, Thm. 2.6]. In particular, corank $H_{\mathrm{et}}^{4}(X, \mathbb{Z}(2))=\operatorname{rank} H_{1}(\Gamma, \mathbb{Z})=$ $\operatorname{rank} H_{\mathrm{et}}^{1}(X, \mathbb{Z})$, which is zero if $X$ has good reduction. In arbitrary dimension, Yoshida shows [36] that corank $H_{\mathrm{et}}^{2 d+2}(X, \mathbb{Z}(d+1))$ is the dimension of the maximal split torus of the Neron model of $A l b_{X}$

Question 4.9. 1) If $X$ has good reduction and $i \leq 2 n$, is $H_{\mathrm{et}}^{i}(X, \mathbb{Z}(n))$ the direct sum of a finitely generated group and a finitely generated torsion free $\mathbb{Z}_{p}$-module (or at least a torsion free $\mathbb{Z}_{(p)}$-module)?

2) If $X$ has good reduction and $i>2 n$, is $H_{\mathrm{et}}^{i}(X, \mathbb{Z}(n))$ the direct sum of a cotorsion group and a cofinitely generated torsion $\mathbb{Z}_{(p)}$-module?

Example 4.8 shows that the answer to the first question is negative if $X$ does not have good reduction.

Question 4.10. Outside the range of the theorem, the groups $H_{\mathrm{et}}^{i}\left(X, \mathbb{Q}_{l} / \mathbb{Z}_{l}(n)\right)$ can have non-zero divisible subgroups, and the groups $H_{\mathrm{et}}^{i}\left(X, \mathbb{Q}_{p} / \mathbb{Z}_{p}(n)\right)$ have at least the same corank as $H_{\mathrm{et}}^{i-1}\left(\bar{X}, \mathbb{Q}_{p} / \mathbb{Z}_{p}\right)$ for all $i$ and $n$ [14, Cor. 11]. Do these groups correspond to a non-torsion subgroup of $H_{\mathrm{et}}^{i}(X, \mathbb{Z}(n))$ or a torsion subgroup of $H_{\mathrm{et}}^{i+1}(X, \mathbb{Z}(n))$ in the short exact coefficient sequence

$$
0 \rightarrow H_{\mathrm{et}}^{i}(X, \mathbb{Z}(n)) \otimes \mathbb{Q}_{l} / \mathbb{Z}_{l} \rightarrow H_{\mathrm{et}}^{i}\left(X, \mathbb{Q}_{l} / \mathbb{Z}_{l}(n)\right) \rightarrow H_{\mathrm{et}}^{i+1}(X, \mathbb{Z}(n))\{l\} \rightarrow 0 \quad ?
$$

Question 4.11. In the examples, the uniquely l-divisible groups appearing have a structure of an $\mathcal{O}_{k}$-module. Is there such a structure in general on a subgroup (of countable index) of $H_{\mathrm{et}}^{i}(X, \mathbb{Z}(n))$ ?

\section{Arithmetic schemes}

Let $j: B \rightarrow C$ be an open subset of the spectrum of the ring of integers of a number field or of a smooth and proper curve over a finite field, and let $S=C-B$ be the set of those places of $C$ not corresponding to a point in $B$, including the infinite places. Let $f: X \rightarrow B$ be smooth and proper, and $d$ the dimension of $X$. For a complex of sheaves $\mathcal{F}$ on $X$ we define cohomology with compact support as the étale cohomology with compact support on $B$ of $R f_{!} \mathcal{F}$, see [16, §3] and [23. II §2]. If $B$ is a curve over a finite field, or if we disregard 2-torsion, then this is the cohomology of $j_{!} R f_{*} \mathcal{F}$ on $C$. By construction, there is an exact sequence [23, Prop. II 2.3]

$$
\cdots \rightarrow H_{c}^{i}(X, \mathcal{F}) \rightarrow H_{\mathrm{et}}^{i}(X, \mathcal{F}) \rightarrow \bigoplus_{v \in S} H_{\mathrm{et}}^{i}\left(X \times_{B} K_{v}, \mathcal{F}\right) \rightarrow \cdots,
$$

for $K_{v}$ the completion of the function field $K$ of $B$ at the place $v$; in case of real fields, it is the Tate-modified cohomology of $R \Gamma_{\text {et }}\left(X_{\mathbb{C}}, \mathcal{F} \cdot\right)$. For properties of Bloch's higher Chow groups on smooth schemes over a Dedekind ring see [7]. The following is the analog of Conjecture 4.1; 
Conjecture 4.12. (Lichtenbaum) If $X$ is regular and proper over the ring of integers of a number field, then the groups $H_{\mathrm{et}}^{i}(X, \mathbb{Z}(n))$ are finitely generated for $i \leq 2 n$, finite for $i=2 n+1$ and of cofinite type for $i \geq 2 n+2$.

Since the Tate-cohomology of $R \Gamma_{\text {et }}\left(X_{\mathbb{C}}, \mathbb{Z}(n)\right)$ is easily seen to be a finite 2group, this is equivalent to the same statement for $H_{c}^{i}(X, \mathbb{Z}(n))$. The following example shows that the analog statement does not hold for arbitrary $B$.

Example 4.13. Let $X=B$ be as above. Then we have the following isomorphisms and exact sequences [23, II Prop. 2.1, 2.6, Cor. 2.11]:

$n=0$ :

$$
\begin{aligned}
& H_{\mathrm{et}}^{0}(B, \mathbb{Z}) \cong \mathbb{Z}, \\
& H_{\mathrm{et}}^{1}(B, \mathbb{Z}) \cong 0, \\
& H_{\mathrm{et}}^{2}(B, \mathbb{Z}) \cong \operatorname{Hom}\left(\pi_{1}^{a b}(B), \mathbb{Q} / \mathbb{Z}\right) .
\end{aligned}
$$

$n=0$, compact support:

$$
\begin{gathered}
0 \rightarrow H_{c}^{0}(B, \mathbb{Z}) \rightarrow \mathbb{Z} \rightarrow \bigoplus_{v \in S} \mathbb{Z} \rightarrow H_{c}^{1}(B, \mathbb{Z}) \rightarrow 0, \\
0 \rightarrow H_{c}^{2}(B, \mathbb{Z}) \rightarrow \pi_{1}^{a b}(B)^{*} \rightarrow \bigoplus_{v \in S} \pi_{1}^{a b}\left(K_{v}\right)^{*} \rightarrow H_{c}^{3}(B, \mathbb{Z}) \rightarrow H_{\mathrm{et}}^{3}(B, \mathbb{Z}) \rightarrow 0 .
\end{gathered}
$$

$n=1$ :

$$
\begin{gathered}
H_{\mathrm{et}}^{1}(B, \mathbb{Z}(1)) \cong \Gamma\left(B, \mathbb{G}_{m}\right)^{\times}, \\
H_{\mathrm{et}}^{2}(B, \mathbb{Z}(1)) \cong \operatorname{Pic}(B), \\
0 \rightarrow H_{\mathrm{et}}^{3}(B, \mathbb{Z}(1)) \rightarrow \bigoplus_{v \in S} \operatorname{Br}\left(K_{v}\right) \rightarrow \mathbb{Q} / \mathbb{Z} \rightarrow H_{\mathrm{et}}^{4}(B, \mathbb{Z}(1)) \rightarrow 0 .
\end{gathered}
$$

The last group vanishes if $B \neq C$; the higher groups are 2-torsion.

$n=1$, compact support:

$$
\begin{aligned}
& H_{c}^{3}(B, \mathbb{Z}(1)) \cong 0, \\
& H_{c}^{4}(B, \mathbb{Z}(1)) \cong \mathbb{Q} / \mathbb{Z}, \\
& H_{c}^{i}(B, \mathbb{Z}(1))=0, \quad i>4 .
\end{aligned}
$$

The following is well-known, but we give a proof for the convenience of the reader: 
Proposition 4.14. If $n \geq 2$ and $B$ is as above, then $H_{\mathrm{et}}^{i}(B, \mathbb{Z}(n))$ is finitely generated.

1) In the number field case, $H_{\mathrm{et}}^{1}(B, \mathbb{Z}(n))$ is finitely generated of rank $r_{1}+r_{2}$ and $r_{2}$ if $n$ is odd and even, respectively, and all other groups are finite.

2) In the function field case, the groups $H_{\mathrm{et}}^{i}(B, \mathbb{Z}(n))$ are finite for all $i$.

Proof. 1) For $B=\operatorname{Spec} \mathcal{O}_{F}$, the case of (non-étale) motivic cohomology $H_{\mathcal{M}}^{i}(B, \mathbb{Z}(n))$ has been treated in [18, Prop. 2.1]. By the Rost-Voevodsky theorem and [7], this gives the result for $i \leq n+1$. Since the groups $H_{\mathrm{et}}^{i}\left(\mathbb{F}_{q}, \mathbb{Z}(n)\right)$ are finite for $n \geq 1$ by Example 4.2, the long exact sequence of [10, Cor. 7.2] shows that the result holds for arbitrary $B$. If $i>n+1 \geq 3$, the same long exact sequence shows that

$$
H_{\mathrm{et}}^{i}(B, \mathbb{Z}(n)) \cong H_{\mathrm{et}}^{i}(K, \mathbb{Z}(n)) \cong H_{\mathrm{et}}^{i-1}(K, \mathbb{Q} / \mathbb{Z}(n))
$$

which is (at most) a finite 2-group.

2) Since $\mathbb{Z} / p(n)=0$ by [11], the groups are uniquely $p$-divisible. Away from $p$, we can use the localization sequence and purity to conclude by the known motivic cohomology of $C$ (Thm. 4.3) and finite fields (Example 4.2).

Example 4.15. There are smooth and proper abelian schemes $X$ of dimension 3 over $B$ such that $H_{\mathrm{et}}^{4}(X, \mathbb{Z}(2))$ contains a divisible torsion group [26].

Lichtenbaum's conjecture suggests the following more general

Conjecture 4.16. The groups $H_{\mathrm{et}}^{i}(X, \mathbb{Z}(n))$ are a direct sum of a finitely generated group and a group of cofinite type.

The analog statement does not hold for $H_{c}^{i}(X, \mathbb{Z}(n))$ if $S$ contains a finite prime $v$; for example the uncountable group $K_{v}^{\times} / K^{\times}$maps onto $H_{c}^{2}(B, \mathbb{Z}(1))$.

To end the paper, we consider an analog of the Tate-Shafarevich group:

Question 4.17. Is the group

$$
S h^{i, n}(X)=\operatorname{ker} H_{\mathrm{et}}^{i}(X, \mathbb{Z}(n)) \rightarrow \bigoplus_{v \in S} H^{i}\left(X \times_{B} K_{v}, \mathbb{Z}(n)\right)
$$

finite if $S$ contains at least one finite prime?

If $S$ does not contain any finite prime, then $\operatorname{Sh}^{4,1}(B) \cong \mathbb{Q} / \mathbb{Z}$, hence the condition is necessary.

Example 4.18. It is easy to see that

$$
S h^{0,0}(X)=S h^{1,0}(X)=S h^{0,1}(X)=S h^{1,1}(X)=0 .
$$

Theorem 4.19. If $f: X \rightarrow B$ is smooth and proper, then $S h^{3,1}(X)$ is finite if and only if $\operatorname{Sh}\left(\mathrm{Pic}_{X_{\eta} / \eta}^{0, r e d}\right)$ is finite. 
Proof. Recall that $\mathbb{Z}(1) \cong \mathbb{G}_{m}[-1]$. Replacing $B$ by $f_{*} \mathcal{O}(X)$, we can assume that $f_{*} \mathbb{G}_{m} \cong \mathbb{G}_{m}$. Indeed, since $X$ is normal, so is $f_{*} \mathcal{O}(X)$, hence is an open subset of the ring of integers of a number field or smooth and proper curve over a finite field again. Moreover, $f^{\prime}: X \rightarrow \operatorname{Spec} f_{*} \mathcal{O}(X)$ is again smooth by SGA I, II Cor. 2.2 , and proper. Consider the spectral sequence

$$
H_{\mathrm{et}}^{s}\left(B, R^{t} f_{*} \mathbb{G}_{m}\right) \Rightarrow H_{\mathrm{et}}^{s+t}\left(X, \mathbb{G}_{m}\right),
$$

and compare it with the local situation:

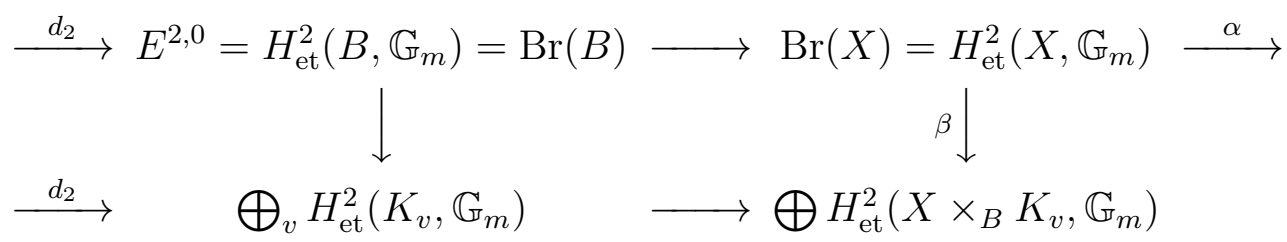

The left vertical map is injective by Example 4.13, and the kernel of the lower horizontal is finite. Indeed, for each $v, X \times_{B} K_{v}$ has a rational point over a finite extension of degree $d$, and then the l-component of the kernel is cyclic of order dividing $d$. We conclude that $\operatorname{ker} \alpha \cap \operatorname{ker} \beta$ is finite, and so it suffices to consider the quotient $\operatorname{Br}_{0}(X)=\operatorname{Br}(X) / \operatorname{im} \operatorname{Br}(B)$. From the spectral sequence we obtain a short exact sequence

$$
0 \rightarrow H_{\mathrm{et}}^{1}\left(B, R^{1} f_{*} \mathbb{G}_{m}\right) \rightarrow \mathrm{Br}_{0}(X) \rightarrow H_{\mathrm{et}}^{0}\left(B, R^{2} f_{*} \mathbb{G}_{m}\right) \stackrel{d_{2}}{\rightarrow} \cdots
$$

Let $g: \eta \rightarrow B$ be the inclusion of the generic point of $B$. Since $\operatorname{Br}(Y) \subseteq \operatorname{Br}(k(Y))$ for any regular integral scheme $Y$, we obtain that $\left.R^{2} f_{*} \mathbb{G}_{m} \subseteq g_{*} R^{2} f_{*} \mathbb{G}_{m}\right|_{\eta}$. Hence

$$
H_{\mathrm{et}}^{0}\left(B, R^{2} f_{*} \mathbb{G}_{m}\right) \subseteq H_{\mathrm{et}}^{0}\left(B,\left.g_{*} R^{2} f_{*} \mathbb{G}_{m}\right|_{\eta}\right)=\operatorname{Br}\left(X \times_{B} \bar{K}\right)^{\operatorname{Gal}(K)},
$$

and the latter is a subgroup of $\left.H_{\mathrm{et}}^{0}\left(K_{v}, R^{2} f_{*} \mathbb{G}_{m}\right) \cong \operatorname{Br}\left(X \times_{B} \bar{K}_{v}\right)\right)^{\operatorname{Gal}\left(K_{v}\right)}$ for any $v$, because $\operatorname{Br}\left(X_{\bar{K}}\right) \subseteq \operatorname{Br}\left(X_{\bar{L}}\right)$ for an extension $\bar{K} \subseteq \bar{L}$ of algebraically closed fields by Proposition 2.4 .

It remains to consider $H_{\text {et }}^{1}(B, P)$ for $P=R^{1} f_{*} \mathbb{G}_{m}$. We claim that there is isomorphism $P \cong g_{*} g^{*} P$. Indeed, the map of stalks at a closed point of $B$ is $H_{\mathrm{et}}^{1}\left(X \times_{B} B_{v}^{s h}, \mathbb{G}_{m}\right) \rightarrow H_{\mathrm{et}}^{1}\left(X \times_{B} K_{v}^{s h}, \mathbb{G}_{m}\right)$, for $B_{v}^{s h}$ the strict henselization of $B$ at $v$ and $K_{v}^{s h}$ its fields of fractions. This map is an isomorphism by the localization sequence of higher Chow groups because $X$ is smooth.

Now consider the reduced connected component $A=\mathrm{Pic}_{X_{\eta} / \eta}^{0, r e d}$ of the Picard scheme $g^{*} P=\operatorname{Pic}_{X_{\eta} / \eta}$ at the generic point. Then $g_{*} A$ is represented by the Neron model $\mathcal{A}$ of $A$. Hence the exact sequence $0 \rightarrow A \rightarrow g^{*} P \rightarrow \operatorname{NS}\left(X_{\eta}\right) \rightarrow 0$ induces an exact sequence $0 \rightarrow \mathcal{A} \rightarrow g_{*} g^{*} P \rightarrow N \rightarrow 0$, where $N$ is a subgroup of $g_{*} \operatorname{NS}\left(X_{\eta}\right)$. The resulting long exact sequence of cohomology groups shows that $H_{\mathrm{et}}^{1}(B, \mathcal{A}) \rightarrow H_{\mathrm{et}}^{1}\left(B, g_{*} g^{*} P\right) \cong H_{\mathrm{et}}^{1}(B, P)$ differ by a finite group. Similarly, 
$H_{\text {et }}^{1}\left(K_{v}, A\right) \rightarrow H_{\text {et }}^{1}\left(K_{v}, g^{*} P\right)$ differ by a finite group. Thus we can conclude by the isomorphism

$$
\operatorname{Sh}(A) \cong \operatorname{ker} H_{\mathrm{et}}^{1}(B, \mathcal{A}) \rightarrow \bigoplus_{v \in S} H_{\mathrm{et}}^{1}\left(K_{v}, A\right)
$$

of [23, II Lemma 5.5].

\section{References}

[1] S. Bloch, H. Esnault, The coniveau filtration and non-divisibility for algebraic cycles. Math. Ann. 304 (1996), no. 2, 303-314.

[2] J.L. Colliot-Thélène, W. RAskind, $K_{2}$-cohomology and the second Chow group. Math. Ann. 270 (1985), no. 2, 165-199.

[3] P. Deligne, Cohomologie étale. Séminaire de Géométrie Algébrique du Bois-Marie SGA 412. Avec la collaboration de J. F. Boutot, A. Grothendieck, L. Illusie et J. L. Verdier. Lecture Notes in Mathematics, Vol. 569. Springer-Verlag, Berlin-New York, 1977. iv+312pp.

[4] K. Fujiwara, A Proof of the Absolute Purity Conjecture (after Gabber), Advanced Studies in Pure Mathematics 36, 2002 Algebraic Geometry 2000, Azumino pp. 153-183.

[5] O. GabBer, Sur la torsion dans la cohomologie $l$-adique d'une variétè. C. R. Acad. Sci. Paris Sér. I Math. 297 (1983), no. 3, 179-182.

[6] T. Geisser, Tate's conjecture, algebraic cycles and rational $K$-theory in characteristic $p$. K-Theory 13 (1998), no. 2, 109-122.

[7] T. Geisser, Motivic cohomology over Dedekind rings. Math. Z. 248 (2004), no. 4, 773-794.

[8] T. Geisser, Weil-etale cohomology, Math. Ann. 330 (2004), 665-692.

[9] T. Geisser, Parshin's conjecture revisited. K-theory and noncommutative geometry, 413-425, EMS Ser. Congr. Rep., Eur. Math. Soc., Zürich, (2008).

[10] T. Geisser, Duality via cycle complexes, Ann. of Math. (2) 172 (2010), no. 2, 1095-1126.

[11] T. Geisser, M. Levine, The K-theory of fields in characteristic $p$. Invent. Math. 139 (2000), no. 3, 459-493. 
[12] M. Gros, N. Suwa Application d'Abel-Jacobi $p$-adique et cycles algébriques. Duke Math. J. 57 (1988), no. 2, 579-613.

[13] B. Kahn, The Geisser-Levine method revisited and algebraic cycles over a finite field. Math. Ann. 324 (2002), no. 3, 581-617.

[14] B. Kahn, Some finiteness results for tale cohomology. J. Number Theory 99 (2003), no. 1, 57-73.

[15] I. Kaplansky, Infinite abelian groups. Revised edition The University of Michigan Press, Ann Arbor, Mich. 1969 vii+95 pp.

[16] K. Kato, A Hasse principle for two dimensional global fields. Journal für die reine und angewandte Mathematik 366, 142-180.

[17] T. Kohrita, Thesis, Nagoya University

[18] M. Kolster, J.W. Sands, Annihilation of motivic cohomology groups in cyclic 2-extensions. Ann. Sci. Math. Québec 32 (2008), no. 2, 175-187.

[19] S. Lichtenbaum, Duality theorems for curves over p-adic fields. Invent. Math. 7 (1969) 120-136.

[20] S. Lichtenbaum, Values of zeta-functions at nonnegative integers. Number theory, Noordwijkerhout 1983 (Noordwijkerhout, 1983), 127138, Lecture Notes in Math., 1068, Springer, Berlin, 1984.

[21] S. Lichtenbuam, The Weil-étale topology on schemes over finite fields, Compositio Math. 141 (2005) 689-702.

[22] J. Milne, Values of zeta functions of varieties over finite fields. Amer. J. Math. 108 (1986), no. 2, 297-360.

[23] J. Milne, Arithmetic duality theorems. Second edition. BookSurge, LLC, Charleston, SC, 2006. viii+339 pp. ISBN: 1-4196-4274-X

[24] J. Murre, Applications of algebraic K-theory to the theory of algebraic cycles. Algebraic geometry, Sitges (Barcelona), 1983, 216-261, Lecture Notes in Math., 1124, Springer, Berlin, 1985.

[25] J. Murre, Algebraic cycles and algebraic aspects of cohomology and K-theory. Algebraic cycles and Hodge theory (Torino, 1993), 93-152, Lecture Notes in Math., 1594, Springer, Berlin, 1994.

[26] A. Rosenschoen, V. Srinivas, Torsion in the Lichtenbaum Chow group of arithmetic schemes. Preprint. 
[27] H. SAito, Abelian varieties attached to cycles of intermediate dimension. Nagoya Math. J. 75 (1979), 95-119.

[28] S. SAito, Class field theory for curves over local fields. J. Number Theory 21 (1985), no. 1, 44-80.

[29] C. Schoen, Some examples of torsion in the Griffiths group. Math. Ann. 293 (1992), no. 4, 651-679.

[30] C. Schoen, On the image of the l-adic Abel-Jacobi map for a variety over the algebraic closure of a finite field. J. Amer. Math. Soc. 12 (1999), no. $3,795-838$.

[31] C. Schoen, On certain exterior product maps of Chow groups. Math. Res. Lett. 7 (2000), no. 2-3, 177-194.

[32] C. Schoen, Complex varieties for which the Chow group mod $n$ is not finite. J. Algebraic Geom. 11 (2002), no. 1, 41-100.

[33] J.P. SERrE, Morphisme universels et varieties d'albanese, Seminaire Chevalley, 1958-1959, Expose 10.

[34] C. SoulÉ, The rank of étale cohomology of varieties over p-adic or number fields. Compositio Math. 53 (1984), no. 1, 113-131.

[35] C. Soulé, Groupes de Chow et K-théorie de variétś sur un corps fini. Math. Ann. 268 (1984), no. 3, 317-345.

[36] T. Yoshida, Finiteness theorems in the class field theory of varieties over local fields. J. Number Theory 101 (2003), no. 1, 138-150.

Department of Mathematics, Rikkyo University, Nishi-IKebukuro, Toshimaku, TOKYo, Japan

E-mail address: geisser@rikkyo.ac.jp 